\title{
Main pulmonary artery enlargement predicts 90 -day readmissions in Chinese COPD patients
}

\author{
Yuqi Zhou", Mohamed Noorul Arafath Thanathi Mohamed Ameen", Wenjuan Li, Dingyun Feng, \\ Hailing Yang, Xiao-Ling Zou, Shaozhu Wu, Tiantuo Zhang \\ Department of Pulmonary and Critical Care Medicine, The Third Affiliated Hospital of Sun Yat-sen University, Guangzhou, China \\ Contributions: (I) Conception and design: T Zhang, Y Zhou, MNA Thanathi Mohamed Ameen; (II) Administrative support: T Zhang; (III) Provision \\ of study materials or patients: T Zhang, Y Zhou; (IV) Collection and assembly of data: MNA Thanathi Mohamed Ameen, W Li, D Feng; (V) Data \\ analysis and interpretation: Y Zhou, MNA Thanathi Mohamed Ameen; (VI) Manuscript writing: All authors (VII) Final approval of manuscript: All \\ author. \\ "These authors contributed equally to this work. \\ Correspondence to: Tiantuo Zhang. Department of Pulmonary and Critical Care Medicine, The Third Affiliated Hospital of Sun Yat-sen University, \\ Guangzhou 510630, China. Email: zhtituli@163.com.
}

Background: Numerous studies have shown pulmonary artery enlargement when measured by chest computed tomography (CT) could predict a worse outcome in chronic obstructive pulmonary disease (COPD) patients. Herein, we studied the prognostic implication of main pulmonary artery diameter (MPAD) in Chinese COPD patients.

Methods: This is an observational case-control study. Patients with 90-day readmissions are case group and those without 90-day readmission are control group. The study comprised of 417 COPD patients who underwent chest CT in their initial admission due to acute exacerbation of COPD (AECOPD). We analyzed their clinical characteristics such as MPAD, arterial blood gas (ABG) results, other chest CT findings and comorbidities to identify the cause of readmission within 90 days.

Results: Median age of our study population is 75 years old, and $79.6 \%$ of them are male. The median MPAD is $2.8 \mathrm{~cm}$ and $80.6 \%$ were also diagnosed with community acquired pneumonia (CAP) in their first admission. The median MPAD in patients with 90-day readmission was $3.1 \mathrm{~cm}$ while patients without 90-day readmission had median $\mathrm{MPAD}$ of $2.8 \mathrm{~cm}$. Through multivariate logistic regression analysis $\mathrm{CAP}(\mathrm{P}=0.019$, OR: 3.105, 95\% CI: 1.203-8.019) and MPAD (P<0.001, OR: 2.898, 95\% CI: 1.824-4.605) were statistically significant. In the second stage of analysis, subgroup of patients diagnosed with CAP and AECOPD (pAECOPD) were analyzed, MPAD remained statistically significant ( $\mathrm{P}<0.001$, OR: 3.490, 95\% CI: 1.929 6.316) and receiver operative characteristic (ROC) curve for pAECOPD patients; area under the curve (AUC) was 0.704 (95\% CI: $0.631-0.778$ ) with a MPAD cut off value of $2.9 \mathrm{~cm}$ (sensitivity $72 \%$, specificity $53 \%$ ).

Conclusions: Enlarged MPAD and pAECOPD in initial admission are independent risk factors for 90 -day readmission. In our pAECOPD patient population, MPAD $>2.9 \mathrm{~cm}$ are at increased risk of 90-day readmission.

Keywords: Chronic obstructive pulmonary disease (COPD); pneumonia; main pulmonary artery diameter (MPAD); main pulmonary artery enlargement

Submitted Mar 07, 2021. Accepted for publication Aug 06, 2021.

doi: $10.21037 /$ jtd-21-344

View this article at: https://dx.doi.org/10.21037/jtd-21-344 


\section{Introduction}

Chronic obstructive pulmonary disease (COPD) is a common, preventable, and treatable disease characterized by persistent respiratory symptoms and airflow limitations (1). It is very frequently complicated by pulmonary hypertension (PHT). Although hypoxia plays a pivotal role, other mechanisms such as acidemia, dynamic pulmonary hyperinflation, parenchymal destruction, pulmonary vascular remodeling, endothelial dysfunction and inflammation also contribute in the pathogenesis of PHT in COPD patients (2-4). These factors are believed to be interdependent and are modulated by genetic factors and maybe confounded by left heart failure, sleep disordered breathing and pulmonary thromboembolism (4). Prevalence of PHT in COPD ranges from $23-90 \%$, depending on the selection of patients and diagnostic criteria (5-8).

PHT must be diagnosed and managed in a timely manner as it can accelerate loss of lung function, increase the risk of exacerbations and death (9-11). The gold standard diagnostic test for PHT is right heart catheterization (RHC), mean pulmonary artery pressure (mPAP) of $\geq 20 \mathrm{mmHg}$ at rest as per the Sixth World Symposium on Pulmonary Hypertension in 2018 (12). Considering the invasive nature of RHC and the complications related to it (hematoma and pneumothorax related to venous access, arrhythmias and episodes of hypotension due to vagal reaction and pulmonary vasoreactivity testing) (2), currently most hospitals and patients in our province resort to other noninvasive means, mainly Doppler echocardiography, to aid in the diagnosis and in the follow up thereafter.

In the past decade, other non-invasive modalities such as chest CT have been extensively studied around the world. Main pulmonary artery diameter (MPAD) enlargement has been proven to correlate with invasive measurements of mPAP obtained by RHC (13-31). Moreover, pulmonary artery enlargement is associated with increased risk of exacerbations of COPD $(17,30,32,33)$ and this particular phenomenon has not been studied thoroughly.

The aim of our study is to find the cut-off point above which risk of exacerbations is markedly increased in our COPD patients diagnosed MPAD through CT measurements. We hope this study will serve as a useful tool for physicians to lower the threshold for conducting further related tests in aiding the early diagnosis, and encourage early management of PHT in our COPD patients, which in turn will drastically change the course of the disease.

We present the following article in accordance with the
STROBE reporting checklist (available at https://dx.doi. org/10.21037/jtd-21-344).

\section{Methods}

This is a retrospective observational case-control study. We analyzed the data of 417 COPD patients from their first hospitalization in the Department of Pulmonary and Critical Care Medicine, The Third Affiliated Hospital of Sun Yat-sen University during the period of January 2007 to July 2015. The case group consist of the COPD patients who were readmitted in our department for the treatment of acute exacerbation of COPD (AECOPD) within 90 days from their discharge day of the first admission and the control group were those who were not readmitted within 90 days from their discharge day of the first admission. Inclusion criteria are as follows: a resident of Guangdong Province, Han Chinese, age above 40, previously diagnosed as COPD through spirometry: post bronchodilator ratio of forced expiratory volume in 1 second to forced vital capacity $\left(\mathrm{FEV}_{1} / \mathrm{FVC}\right)<0.7$, first admission and the readmission in 90 days (if applicable) must be for management of the AECOPD, availability of Chest CT data from their first admission, arterial blood gas (ABG) data from the first day of their first admission and data regarding comorbidities such as hypertension, congestive heart failure and interstitial pneumonia.

Community acquired pneumonia (CAP) was diagnosed according to clinical practice guidelines by the Chinese Thoracic Society, Chinese Medical Association. The diagnostic criteria are as follows: (I) onset in community. (II) Relevant clinical manifestations of pneumonia: (i) new onset of cough or expectoration, or aggravation of existing symptoms of respiratory tract diseases, with or without purulent sputum, chest pain, dyspnea, or hemoptysis; (ii) fever; (iii) signs of pulmonary consolidation and/or moist rales; (iv) peripheral white blood cell count (WBC) $>10 \times 10^{9} / \mathrm{L}$ or $<4 \times 10^{9} / \mathrm{L}$, with or without a left shift. (III) Chest radiograph showing new patchy infiltrates, lobar or segmental consolidation, ground-glass opacities, or interstitial changes, with or without pleural effusion. Clinical diagnosis can be established if a patient satisfies Criterion I, Criterion III and any one condition of Criterion II and meanwhile, tuberculosis, pulmonary tumor, noninfectious interstitial lung disease, pulmonary edema, atelectasis, pulmonary embolism, pulmonary eosinophilia and pulmonary vasculitis are all excluded. Although the criteria quoted by the Chinese medical association quoted 


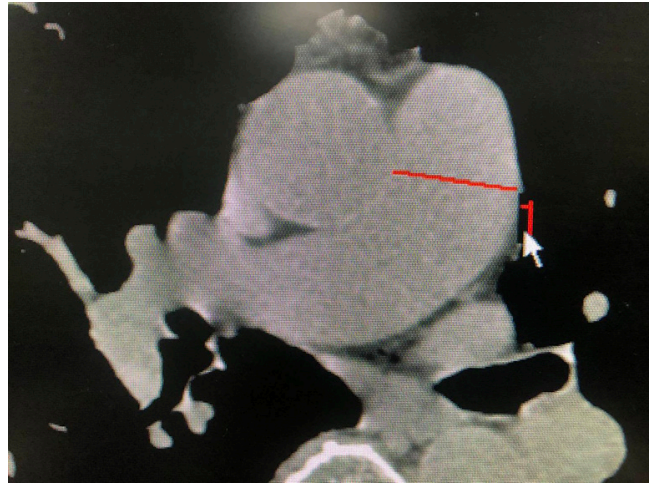

Figure 1 The MPAD was measured on axial plane at the tubular site, widest diameter perpendicular to the short axis of pulmonary artery. MPAD, main pulmonary artery diameter.

chest radiograph abnormalities, in this study chest CT was used to aid in the diagnosis.

Patients' demographic data, number of hospitalization days, ABG analysis results from the first day of first admission in our hospital, presence of comorbidities such as hypertension, congestive heart failure, interstitial pneumonia and data from the contrast or non-contrast chest $\mathrm{CT}$ such as pulmonary artery diameter, interstitial pneumonic changes and characteristic pneumonic changes from the first hospitalization were analyzed to predict worse prognosis, i.e., a particular patient being readmitted within 90 days. Exacerbation was defined as aggravation of at least one-third respiratory symptoms (dyspnea, cough, or sputum purulence) that require hospitalization and 90-day readmission defined as rehospitalization within 90-day from the day of discharge of the first admission (both admissions must be for the management of AECOPD).

Chest CT in our study was performed on patients during their first admissions within the first three days, patient in supine position and images taken in inspiration were used in this study. Two pulmonologists independently measured the MPAD and average of it was used in this study. The MPAD was measured on axial plane at the tubular site, widest diameter perpendicular to the short axis of pulmonary artery (Figure 1). Moreover, the interobserver reliability was assessed by calculating intraclass correlation coefficients (ICCs). Other data of significant pneumonic changes and interstitial pneumonic changes were also noted down. We were only able to retrieve height and weight of 96 patients from our records and mean and standard deviation of body mass index (BMI) and body surface area (BSA) were calculated from these data.
All data stated above was retrieved from medical case record database software (His software) in our hospital. The study was conducted in accordance with the Declaration of Helsinki (as revised in 2013). The study was reviewed and approved by the Medical Ethics Committee of the Third Affiliated Hospital of Sun Yat-sen University \{[2020]-02186-01\}. The need for patient consent was waived as the study was retrospective in nature and no invasive procedures were conducted as part of this study.

The statistical analysis was done in IBM SPSS ver25.0 software. We began analyzing our baseline data and clinical characteristics with Mann Whitney $U$ test for continuous variables (as they did not follow normal distribution, median and interquartile range were used to describe them) and chisquare test for categorical variables(percentages were used for description), then proceeding onto univariate logistic regression, those variables with $P$ value of less than 0.25 was further analyzed using the multivariate logistic regression technique with backward logistic regression option. In all analysis, $\mathrm{P}$ value of less than 0.05 is considered to be statistically significant. Our first stage of analysis included all AECOPD patients (who were also diagnosed with or without CAP). Second stage of analysis only included AECOPD patients who were also diagnosed with CAP (pAECOPD) and our third stage of analysis only included AECOPD patients without CAP (npAECOPD). Finally, a receiver operative characteristic (ROC) curve was plotted in order to declare the cutoff point of MPAD above which our patients are at increased risk of 90-day readmissions with AECOPD.

\section{Results}

The median age of our study population is 75 years old, and $79.6 \%$ of them are male. The median MPAD is $2.8 \mathrm{~cm}$ and $80.6 \%$ were also diagnosed with CAP in their first admission. Table 1 shows baseline characteristics of all AECOPD patients in our study. The median MPAD in patients with 90-day readmission was $3.1 \mathrm{~cm}$ while patients without 90-day readmission had median MPAD of $2.8 \mathrm{~cm}$. Among the 62 patients with readmissions, 57 (92.0\%) of them had also been diagnosed with CAP. Table 2 presents baseline characteristics when pAECOPD and npAECOPD patients were separately analyzed to assess the bivariate association with 90-day readmission. The median MPAD among the pAECOPD patients $2.9 \mathrm{~cm}$ while it was $2.7 \mathrm{~cm}$ in npAECOPD group. For all the MPAD measurements performed by both pulmonologists, inter-rater reliability 
Table 1 Baseline data and clinical characteristics

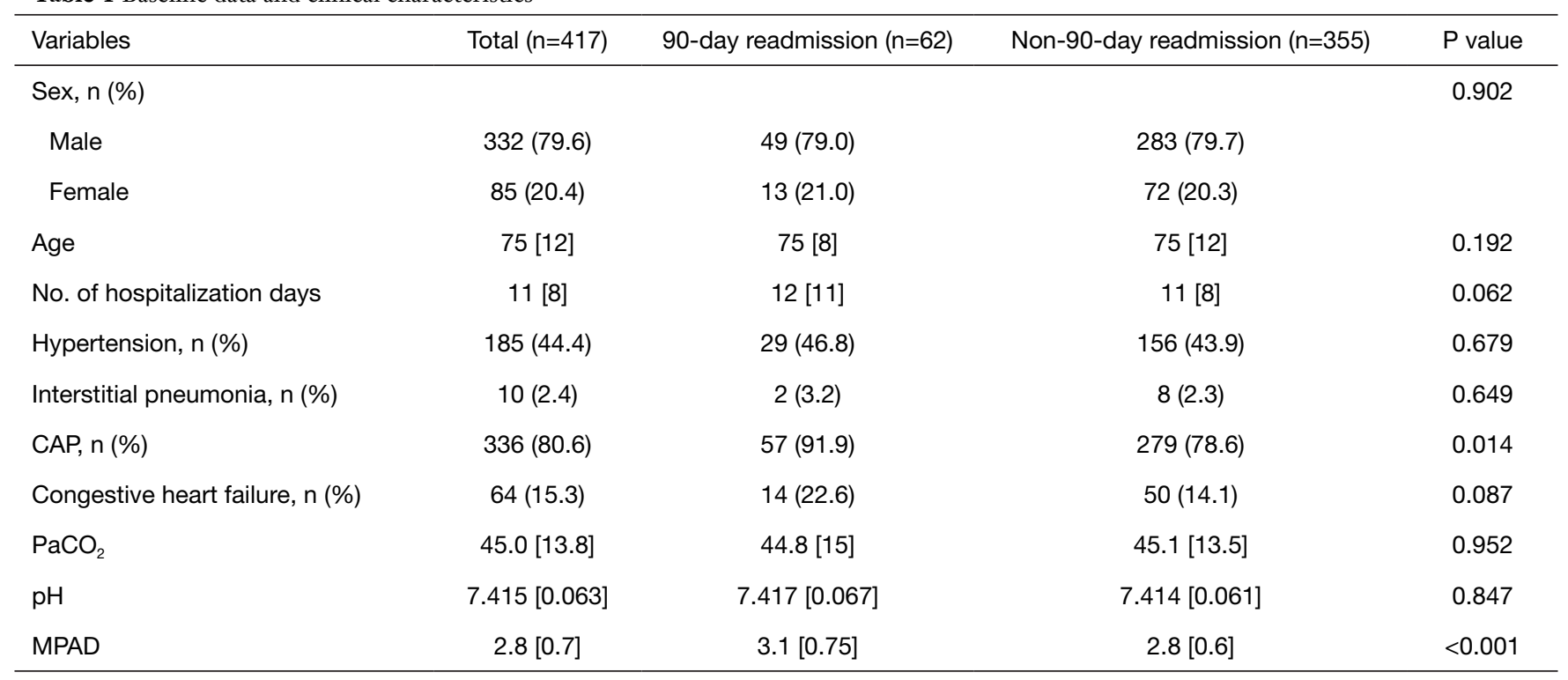

CAP, community acquired pneumonia; MPAD, main pulmonary artery diameter.

Table 2 Baseline data and clinical characteristics of pAECOPD and npAECOPD patients

\begin{tabular}{|c|c|c|c|c|}
\hline Variables & \multicolumn{2}{|c|}{ pAECOPD } & \multicolumn{2}{|c|}{ npAECOPD } \\
\hline Sex, n (\%) & & 0.987 & & 0.871 \\
\hline Male & $265(78.9)$ & & $67(82.7)$ & \\
\hline Female & $71(21.1)$ & & $14(17.3)$ & \\
\hline No. of hospitalization days & $11[8]$ & 0.147 & $10[7]$ & 0.608 \\
\hline Hypertension, n (\%) & $146(43.5)$ & 0.513 & $39(48.1)$ & 0.705 \\
\hline Interstitial pneumonia, n (\%) & $5(1.5)$ & 0.859 & $5(6.2)$ & 0.275 \\
\hline Congestive heart failure, $\mathrm{n}(\%)$ & $51(15.2)$ & 0.078 & $13(16.0)$ & 0.869 \\
\hline MPAD & $2.9[0.7]$ & $<0.001$ & $2.7[0.65]$ & 0.798 \\
\hline
\end{tabular}

PAECOPD, diagnosed CAP and AECOPD; npAECOPD, diagnosed only AECOPD; MPAD, main pulmonary artery diameter; CAP, community acquired pneumonia; AECOPD, acute exacerbation of chronic obstructive pulmonary disease.

was assessed by calculating ICCs which suggested very strong correlation with an ICCs of 0.99. In regard to height and weight of the study population, only records of 96 out of the 417 patients included in our study were retrievable from our database. Mean BMI and BSA of our study group are $20.10 \pm 3.19 \mathrm{~kg} / \mathrm{m}^{2}$ and $1.54 \pm 0.17 \mathrm{~m}^{2}$.
Univariate and multivariate logistic regression analysis were done thrice in this study to identify the independent risk factors for 90-day readmission in different subgroup of population in our study. First stage analysis, all AECOPD patients (Tables 3,4), second stage analysis, only pAECOPD patients (Tables 5,6) and third stage analysis, npAECOPD 
Table 3 Univariate regression analysis of all AECOPD patients

\begin{tabular}{|c|c|c|c|}
\hline Variables & $P$ value & OR & $95 \% \mathrm{Cl}$ \\
\hline Age & 0.091 & 1.027 & $0.996-1.060$ \\
\hline No. of hospitalization days & 0.596 & 1.005 & $0.987-1.024$ \\
\hline Hypertension & 0.679 & 1.121 & $0.653-1.926$ \\
\hline CAP & 0.019 & 3.105 & $1.203-8.019$ \\
\hline Congestive heart failure & 0.090 & 1.779 & $0.914-3.464$ \\
\hline $\mathrm{PaCO}_{2}$ & 0.153 & 1.015 & $0.995-1.035$ \\
\hline $\mathrm{pH}$ & 0.265 & 0.095 & $0.002-5.945$ \\
\hline
\end{tabular}

AECOPD, acute exacerbation of chronic obstructive pulmonary disease; CAP, community acquired pneumonia; MPAD, main pulmonary artery diameter.

Table 4 Multivariate regression analysis of all AECOPD patients

\begin{tabular}{llll}
\hline Variables & P value & OR & 95\% Cl \\
\hline MPAD & $<0.001$ & 2.945 & $1.808-4.798$ \\
CAP & 0.037 & 2.819 & $1.063-7.474$ \\
Congestive heart failure & 0.179 & 1.617 & $0.802-3.261$ \\
$\mathrm{Age}$ & 0.208 & 1.022 & $0.988-1.057$ \\
$\mathrm{PaCO}_{2}$ & 0.206 & 1.013 & $0.993-1.034$ \\
\hline
\end{tabular}

AECOPD, acute exacerbation of chronic obstructive pulmonary disease; MPAD, main pulmonary artery diameter; CAP, community acquired pneumonia.

Table 5 Univariate logistic regression of pAECOPD patients

\begin{tabular}{lccc}
\hline Variables & P value & OR & $95 \% \mathrm{Cl}$ \\
\hline Sex & 0.987 & 1.006 & $0.500-2.022$ \\
Age & 0.150 & 1.024 & $0.991-1.059$ \\
No. of hospitalization days & 0.764 & 1.003 & $0.984-1.022$ \\
Hypertension & 0.513 & 1.210 & $0.683-2.143$ \\
Interstitial pneumonia & 0.856 & 1.228 & $0.135-11.192$ \\
Congestive heart failure & 0.082 & 0.082 & $0.924-3.800$ \\
PaCO & & 1.011 & $0.990-1.032$ \\
PH & 0.315 & 0.294 & $0.004-23.680$ \\
MPAD & 0.585 & 3.498 & $1.939-6.311$ \\
\hline
\end{tabular}

PAECOPD, diagnosed CAP and AECOPD; MPAD, main pulmonary artery diameter; CAP, community acquired pneumonia; AECOPD, acute exacerbation of chronic obstructive pulmonary disease. 
Table 6 Multivariate logistic regression of pAECOPD patients

\begin{tabular}{llll}
\hline Variables & P value & OR & \multicolumn{1}{c}{$95 \% \mathrm{Cl}$} \\
\hline MPAD & $<0.001$ & 3.490 & $1.939-6.316$ \\
Age & 0.197 & 1.024 & $0.988-1.06$ \\
Congestive heart failure & 0.179 & 1.664 & $0.792-3.494$ \\
\hline
\end{tabular}

pAECOPD, diagnosed CAP and AECOPD; MPAD, main pulmonary artery diameter; CAP, community acquired pneumonia; AECOPD, acute exacerbation of chronic obstructive pulmonary disease.

Table 7 Univariate regression of nPAECOPD patients

\begin{tabular}{lccc}
\hline Variables & P value & OR & \multicolumn{1}{c}{$95 \% \mathrm{Cl}$} \\
\hline Sex & 0.868 & 0.825 & $0.085-7.999$ \\
Age & 0.654 & 1.025 & $0.921-1.140$ \\
No. of hospitalization days & 0.998 & 1.000 & $0.889-1.126$ \\
Hypertension & 0.708 & 0.703 & $0.111-4.446$ \\
Interstitial pneumonia & 0.222 & 4.500 & $0.404-50.180$ \\
Congestive heart failure & 0.804 & 1.333 & $0.137-12.989$ \\
PaCO & 0.156 & 1.050 & $0.981-1.123$ \\
pH & 0.148 & $<0.001$ & $0.001-35.623$ \\
MPAD & 0.973 & 0.972 & $0.193-4.899$ \\
\hline
\end{tabular}

npAECOPD, diagnosed only AECOPD; MPAD, main pulmonary artery diameter; CAP, community acquired pneumonia; AECOPD, acute exacerbation of chronic obstructive pulmonary disease.

Table 8 Multiple regression of nPAECOPD patients

\begin{tabular}{lccc}
\hline Variables & P value & OR & $95 \% \mathrm{Cl}$ \\
\hline $\mathrm{PaCO}_{2}$ & 0.458 & 1.030 & $0.953-1.112$ \\
Interstitial pneumonia & 0.092 & 10.025 & $0.685-146.630$ \\
$\mathrm{pH}$ & 0.074 & $<0.001$ & $0.001-3.513$ \\
\hline
\end{tabular}

npAECOPD, diagnosed only AECOPD; CAP, community acquired pneumonia; AECOPD, acute exacerbation of chronic obstructive pulmonary disease.

patients (Tables 7,8). During the first stage analysis, through multiple regression analysis, we were able to establish that CAP ( $\mathrm{P}=0.019$, OR: 3.105, 95\% CI: 1.203-8.019) and MPAD ( $\mathrm{P}<0.001$, OR: 2.898, 95\% CI: 1.824-4.605) are independent risk factors for 90 -day readmission. In the second stage of analysis, multivariate logistic regression showed MPAD ( $\mathrm{P}<0.001$, OR: 3.490, 95\% CI: 1.929-6.316) was statistically significant. In the third stage of analysis, univariate logistic regression results were $\mathrm{PaCO}_{2}$, interstitial pneumonia and $\mathrm{pH}$ had $\mathrm{P}<0.25$ but when multivariate regression was done none of the variables were statistically significant. We plotted a ROC curve for pAECOPD patients; area under the curve (AUC) was 0.704 (95\% CI: 0.631-0.778) with a MPAD cut off value of $2.9 \mathrm{~cm}$ (sensitivity $72 \%$, specificity $53 \%$ ).

\section{Discussion}

Through our study we were able to establish that existence of concomitant diagnosis of CAP and main pulmonary artery enlargement in initial admission are independent risk factors for readmission within 90 days among our AECOPD 
patients. Among our pAECOPD patients, MPAD >2.9 cm can predict a 90 -day readmission with a sensitivity of $72 \%$ and a specificity of $53 \%$.

Three hundred and thirty-six out of 417 (80.6\%) of our overall study population and 57 out of 62 (91.9\%) cases who had 90-day readmission were diagnosed with CAP in their initial hospitalization. This is one of the reasons why we designed our study to analyze pAECOPD (stage 2 analysis) and npAECOPD (stage 3 analysis) patient subgroups separately to avoid bias in our study. We believe the high rate of CAP among our AECOPD patients is mainly due the following reasons. Firstly, Guangdong Province is an industrial area with tens of thousands of factories and this has led to air pollution which is far more severe than the developed countries. A study by Steer $e t$ al. in the United Kingdom showed that among his AECOPD patients, only $32.5 \%$ had a concomitant diagnosis of CAP by chest radiograph (34). Moreover, in a study by Claessens et al., $33 \%$ of their patients who did not have parenchymal infiltrates in chest radiograph, had chest CT parenchymal infiltrates (35). In our study, all our patients had undergone chest $\mathrm{CT}$ which is more sensitive and specific in identifying pneumonic infiltrates which also explains for the higher proportion of pAECOPD patients.

There have been contradictory study results regarding readmission rates of $\mathrm{pAECOPD}$ and $\mathrm{npAECOPD}$ subgroups. Some researchers have concluded that pAECOPD cause less readmissions (36) while others have concluded there is no difference in readmission rates between pAECOPD and npAECOPD patients (37). Meanwhile, in our study population, evidence suggests that patients diagnosed pAECOPD in initial admission is an important risk factor for 90 -day readmission $(\mathrm{P}=0.019$, OR: 3.105, 95\% CI: 1.203-8.019).

Apart from hypoxic vasoconstriction, studies have shown that multiple pathways of inflammation also contribute to the development of pulmonary vascular disease and PHT in COPD. C-reactive protein (CRP), tumor necrosis factoralpha (TNF- $\alpha$ ), monocyte chemoattractant protein-1, soluble intercellular adhesion molecule-1, platelet-derived growth factor and interleukin-6 (IL-6) are among the inflammatory markers which have been believed to be involved in pathophysiology of PHT in COPD patients. Furthermore, circulating IL-6 level correlates with pulmonary artery pressures $(38,39)$. COPD patients in general have higher IL-6 levels and those with COPD and PHT have even more higher circulating IL-6 levels (39).

Huerta $e t a l$. investigated the inflammatory markers among pAECOPD and npAECOPD patients and found that pAECOPD patients had more powerful biologic signaling, mediated by several biomarkers [CRP, procalcitonin (PCT), TNF- $\alpha$, and IL-6] (36). This is the second reason we chose to further analyze pAECOPD (stage 2 analysis) and npAECOPD subgroups separately in an attempt to control any possible bias, further improving the accuracy and strength of our meticulously designed study, Other researches which aimed to study relation between pulmonary artery diameter and PHT (13-31) and those researches which studied pulmonary artery enlargement and acute exacerbations of COPD $(30,32,33)$ failed to address and take the pathobiologic difference between pAECOPD and npAECOPD as explained above. We encourage future researchers to consider this aspect and strongly believe this approach will produce a more reliable result.

Many researchers have measured the widest MPAD diameter on the axial image at the bifurcation site [method 2 mentioned in Mahammedi et al. (15)] while MPAD diameter at the tubular site have been only considered by few researchers $(21,32)$. It is difficult to determine the bifurcation site due to the morphologically complex shape and axis of the MPAD and its branches. CT MPAD measurement at the tubular site will minimize the intra observer and interobserver variability. We chose to include only MPAD in our study instead of prioritizing MPAD: ascending aorta (AA) diameter for the following reasons; firstly, our study consisted of both contrast and non-contrast chest CT, thickness of the aortic wall will be significantly thicker in comparison to pulmonary arterial wall and aortic wall thickness may vary among individuals leading to overestimated readings especially in the non-contrast chest CT, according to the meta-analysis study published by Shen et al., MPAD had higher sensitivity and specificity for the diagnosis of PHT (14), and finally, after the age of 50, diameter of aorta often dilates rendering MPAD: AA ratio a less reliable tool (19).

de-Torres et al. conducted an observational study (consisted of 188 COPD patients) concluded that having a $M P A D>30 \mathrm{~mm}$ on chest $\mathrm{CT}$ as a powerful and independent predictor of survival among their patient population (40). This was contradictory as most related MPAD related researches suggested that the pulmonary artery enlargement correlates with mPAP and it aids with the diagnosis of PHT, exacerbations and worse outcomes. In another prospective observational study, Boerrigter et al. followed up 51 patients diagnosed with pulmonary arterial hypertension and found that in all the patients in whom the pressure was 
higher than initial measurements, the pulmonary artery diameter had increased. However, if the pressure was lower than initial measurements, the majority of this group's pulmonary artery diameters still increased (41). Another prospective study conducted by Akagi et al. also concluded that pulmonary artery dilatation progressed in patients with a pulmonary artery aneurysm $(\mathrm{MPAD}>4 \mathrm{~cm})$ despite treatment of PHT and the progression of pulmonary artery dilatation is independent of reduction of pulmonary artery pressure by PHT treatment (42).

From careful consideration of the three studies mentioned above, it is safe to conclude that CT measurements of MPAD is only reliable in the early stage of the disease where it can be used as diagnostic tool. Therefore, our study only included the data of patients from the very first admission (for AECOPD management) in our department.

Our study was designed carefully taking into account many of the factors as discussed above which we believe have greatly improved the precision of our results. To the best of our knowledge, our study is the first to have found cut off point of MPAD (short axis widest diameter at the tubular site) which can differentiate those with higher risk for 90-day readmission among our pAECOPD patients in our province. This will definitely be a useful and readily available tool for physicians in detecting high risk patients and further gives a chance to incorporate more active management approach for these patients. Chest CT is available in most tertiary hospitals in our province and MPAD readings at tubular site is very easy to learn and can easily be reproduced.

Although our study has a monocentric design, our sample size is comparatively large and our COPD patients are from all around Guangdong Province as our hospital is a tertiary hospital which is renowned for its COPD care in Guangdong Province. Monocentric studies do come with its own advantages; we had the same clinical approach from our team, same team of pulmonologists and radiologists provided the services, we also limited the duration of our study (8.5 years) to prevent fluctuations in care provided due to differences in technology or the team of doctors.

Hypoxia is one of the important factors which independently affects the pulmonary vasoconstriction (4) which in turn may cause fluctuations in the pulmonary artery diameter, we were not able to include this important variable to our analysis as most patients were on supplemental oxygen whilst the ABG arterial blood samples were taken. Our study being a retrospective in nature, some factors like that discussed above could not be controlled.
A significant amount of our patient population had hypertension (44.4\%) and among them some may have been on angiotensin receptor blockers, angiotensin converting enzyme inhibitors, other vasodilator treatments, or if our patients had been prescribed azithromycin in the outpatient setting for its immunomodulatory effects and reducing the risk of exacerbations. This may have affected the precision of our results.

\section{Conclusions}

Enlarged MPAD and pAECOPD in initial admission are independent risk factors for 90-day readmission. In our pAECOPD patient population, MPAD $>2.9 \mathrm{~cm}$ are at increased risk of 90-day readmission. Nevertheless, a multicenter study with large patient population and similar study design is needed to establish an even more accurate cut off value.

\section{Acknowledgments}

Funding: This work was supported by Guangzhou Science and Technology Project Fund (201709010040) and Guangdong Medical Research Foundation (No. A2019062).

\section{Footnote}

Reporting Checklist: The authors have completed the STROBE reporting checklist. Available at https://dx.doi. org/10.21037/jtd-21-344

Data Sharing Statement: Available at https://dx.doi. org/10.21037/jtd-21-344

Conflicts of Interest: All authors have completed the ICMJE uniform disclosure form (available at https://dx.doi. org/10.21037/jtd-21-344). All authors report funding from Guangzhou Science and Technology Project Fund (201709010040) and Guangdong Medical Research Foundation (No. A2019062).

Ethical Statement: The authors are accountable for all aspects of the work in ensuring that questions related to the accuracy or integrity of any part of the work are appropriately investigated and resolved. The study was conducted in accordance with the Declaration of Helsinki (as revised in 2013). The study was reviewed and approved by The Medical Ethics Committee of the Third Affiliated 
Hospital of Sun Yat-sen University \{[2020]-02-186-01\}. The need for patient consent was waived as the study was retrospective in nature and no invasive procedures were conducted as part of this study.

Open Access Statement: This is an Open Access article distributed in accordance with the Creative Commons Attribution-NonCommercial-NoDerivs 4.0 International License (CC BY-NC-ND 4.0), which permits the noncommercial replication and distribution of the article with the strict proviso that no changes or edits are made and the original work is properly cited (including links to both the formal publication through the relevant DOI and the license). See: https://creativecommons.org/licenses/by-nc-nd/4.0/.

\section{References}

1. Global strategy for diagnosis, management and prevention of chronic obstructive pulmonary disease: 2020 report. Available online: https://goldcopd.org/wp-content/ uploads/2019/12/GOLD-2020-FINAL-ver1.2-03Dec19_ WMV.pdf

2. Coste F, Benlala I, Dournes G, et al. Assessing pulmonary hypertension in COPD. Is there a role for computed tomography? Int J Chron Obstruct Pulmon Dis 2019;14:2065-79.

3. Wells JM, Dransfield MT. Pathophysiology and clinical implications of pulmonary arterial enlargement in COPD. Int J Chron Obstruct Pulmon Dis 2013;8:509-21.

4. Wrobel JP, Thompson BR, Williams TJ. Mechanisms of pulmonary hypertension in chronic obstructive pulmonary disease: a pathophysiologic review. J Heart Lung Transplant 2012;31:557-64.

5. Sims MW, Margolis DJ, Localio AR, et al. Impact of pulmonary artery pressure on exercise function in severe COPD. Chest 2009;136:412-9.

6. Scharf SM, Iqbal M, Keller C, et al. Hemodynamic characterization of patients with severe emphysema. Am J Respir Crit Care Med 2002;166:314-22.

7. Minai OA, Chaouat A, Adnot S. Pulmonary hypertension in COPD: epidemiology, significance, and management: pulmonary vascular disease: the global perspective. Chest 2010;137:39S-51S.

8. Hoeper MM, Barberà JA, Channick RN, et al. Diagnosis, assessment, and treatment of non-pulmonary arterial hypertension pulmonary hypertension. J Am Coll Cardiol 2009;54:S85-96.

9. Weitzenblum E, Hirth C, Ducolone A, et al. Prognostic value of pulmonary artery pressure in chronic obstructive pulmonary disease. Thorax 1981;36:752-8.

10. Hurdman J, Condliffe R, Elliot CA, et al. ASPIRE registry: assessing the Spectrum of Pulmonary hypertension Identified at a REferral centre. Eur Respir J 2012;39:945-55.

11. Kessler R, Faller M, Weitzenblum E, et al. "Natural history" of pulmonary hypertension in a series of 131 patients with chronic obstructive lung disease. Am J Respir Crit Care Med 2001;164:219-24.

12. Condon DF, Nickel NP, Anderson R, et al. The 6th World Symposium on Pulmonary Hypertension: what's old is new. F1000Res 2019;8:F1000 Faculty Rev-888.

13. Kuriyama K, Gamsu G, Stern RG, et al. CT-determined pulmonary artery diameters in predicting pulmonary hypertension. Invest Radiol 1984;19:16-22.

14. Shen Y, Wan C, Tian P, et al. CT-base pulmonary artery measurement in the detection of pulmonary hypertension: a meta-analysis and systematic review. Medicine (Baltimore) 2014;93:e256.

15. Mahammedi A, Oshmyansky A, Hassoun PM, et al. Pulmonary artery measurements in pulmonary hypertension: the role of computed tomography. J Thorac Imaging 2013;28:96-103.

16. Tan RT, Kuzo R, Goodman LR, et al. Utility of CT scan evaluation for predicting pulmonary hypertension in patients with parenchymal lung disease. Medical College of Wisconsin Lung Transplant Group. Chest 1998;113:1250-6.

17. Dornia C, Lange TJ, Behrens G, et al. Multidetector computed tomography for detection and characterization of pulmonary hypertension in consideration of $\mathrm{WHO}$ classification. J Comput Assist Tomogr 2012;36:175-80.

18. Lange TJ, Dornia C, Stiefel J, et al. Increased pulmonary artery diameter on chest computed tomography can predict borderline pulmonary hypertension. Pulm Circ 2013;3:363-8.

19. Ng CS, Wells AU, Padley SP. A CT sign of chronic pulmonary arterial hypertension: the ratio of main pulmonary artery to aortic diameter. J Thorac Imaging 1999;14:270-8.

20. Truong QA, Bhatia HS, Szymonifka J, et al. A fourtier classification system of pulmonary artery metrics on computed tomography for the diagnosis and prognosis of pulmonary hypertension. J Cardiovasc Comput Tomogr 2018;12:60-6.

21. Chan AL, Juarez MM, Shelton DK, et al. Novel computed tomographic chest metrics to detect pulmonary hypertension. BMC Med Imaging 2011;11:7. 
22. Guthaner DF, Wexler L, Harell G. CT demonstration of cardiac structures. AJR Am J Roentgenol 1979;133:75-81.

23. Kam JC, Pi J, Doraiswamy V, et al. CT scanning in the evaluation of pulmonary hypertension. Lung 2013;191:321-6.

24. Shin S, King CS, Brown AW, et al. Pulmonary artery size as a predictor of pulmonary hypertension and outcomes in patients with chronic obstructive pulmonary disease. Respir Med 2014;108:1626-32.

25. Burger IA, Husmann L, Herzog BA, et al. Main pulmonary artery diameter from attenuation correction CT scans in cardiac SPECT accurately predicts pulmonary hypertension. J Nucl Cardiol 2011;18:634-41.

26. Sanal S, Aronow WS, Ravipati G, et al. Prediction of moderate or severe pulmonary hypertension by main pulmonary artery diameter and main pulmonary artery diameter/ascending aorta diameter in pulmonary embolism. Cardiol Rev 2006;14:213-4.

27. Zisman DA, Karlamangla AS, Ross DJ, et al. Highresolution chest CT findings do not predict the presence of pulmonary hypertension in advanced idiopathic pulmonary fibrosis. Chest 2007;132:773-9.

28. Shin S, King CS, Puri N, et al. Pulmonary artery size as a predictor of outcomes in idiopathic pulmonary fibrosis. Eur Respir J 2016;47:1445-51.

29. Iyer AS, Wells JM, Vishin S, et al. CT scan-measured pulmonary artery to aorta ratio and echocardiography for detecting pulmonary hypertension in severe COPD. Chest 2014;145:824-32.

30. Wells JM, Washko GR, Han MK, et al. Pulmonary arterial enlargement and acute exacerbations of COPD. N Engl J Med 2012;367:913-21.

31. Devaraj A, Wells AU, Meister MG, et al. Detection of pulmonary hypertension with multidetector CT and echocardiography alone and in combination. Radiology 2010;254:609-16.

32. Rho JY, Lynch DA, Suh YJ, et al. CT measurements of central pulmonary vasculature as predictors of severe exacerbation in COPD. Medicine (Baltimore)

Cite this article as: Zhou Y, Thanathi Mohamed Ameen MNA, Li W, Feng D, Yang H, Zou XL, Wu S, Zhang T. Main pulmonary artery enlargement predicts 90 -day readmissions in Chinese COPD patients. J Thorac Dis 2021;13(10):5731-5740. doi: $10.21037 /$ jtd-21-344 2018;97:e9542.

33. Chung KS, Kim YS, Kim SK, et al. Functional and prognostic implications of the main pulmonary artery diameter to aorta diameter ratio from chest computed tomography in Korean COPD patients. PLoS One 2016;11:e0154584.

34. Steer J, Norman EM, Afolabi OA, et al. Dyspnoea severity and pneumonia as predictors of in-hospital mortality and early readmission in acute exacerbations of COPD. Thorax 2012;67:117-21.

35. Claessens YE, Debray MP, Tubach F, et al. Early chest computed tomography scan to assist diagnosis and guide treatment decision for suspected community-acquired pneumonia. Am J Respir Crit Care Med 2015;192:974-82.

36. Huerta A, Crisafulli E, Menéndez R, et al. Pneumonic and nonpneumonic exacerbations of COPD: inflammatory response and clinical characteristics. Chest 2013;144:1134-42.

37. Husebø GR, Grønseth R, Lehmann S, et al. Pneumonia vs non-pneumonia in COPD exacerbations-different risks? Eur Respir J 2018;52:PA2277.

38. Eddahibi S, Chaouat A, Tu L, et al. Interleukin-6 gene polymorphism confers susceptibility to pulmonary hypertension in chronic obstructive pulmonary disease. Proc Am Thorac Soc 2006;3:475-6.

39. Chaouat A, Savale L, Chouaid C, et al. Role for interleukin-6 in COPD-related pulmonary hypertension. Chest 2009;136:678-87.

40. de-Torres JP, Ezponda A, Alcaide AB, et al. Pulmonary arterial enlargement predicts long-term survival in COPD patients. PLoS One 2018;13:e0195640.

41. Boerrigter B, Mauritz GJ, Marcus JT, et al. Progressive dilatation of the main pulmonary artery is a characteristic of pulmonary arterial hypertension and is not related to changes in pressure. Chest 2010;138:1395-401.

42. Akagi S, Nakamura K, Sarashina T, et al. Progression of pulmonary artery dilatation in patients with pulmonary hypertension coexisting with a pulmonary artery aneurysm. J Cardiol 2018;71:517-22. 